\title{
Lidil
}

Revue de linguistique et de didactique des langues

\section{Phrases complexes et maturité syntaxique : une comparaison entre des écrits d'élèves de 13 et}

\section{6 ans}

Complex Sentences and Syntactic Maturity: A Comparison between 8th and 11th Grade Students

Marie-Claude Boivin, Katrine Roussel et Reine Pinsonneault

\section{OpenEdition}

Journals

Édition électronique

URL : http://journals.openedition.org/lidil/4206

DOI : 10.4000/lidil.4206

ISSN : $1960-6052$

Éditeur

UGA Éditions/Université Grenoble Alpes

Édition imprimée

ISBN : 978-2-37747-002-0

ISSN : $1146-6480$

\section{Référence électronique}

Marie-Claude Boivin, Katrine Roussel et Reine Pinsonneault, «Phrases complexes et maturité syntaxique : une comparaison entre des écrits d'élèves de 13 et 16 ans », Lidil [En ligne], 55 | 2017, mis en ligne le 02 mai 2017, consulté le 19 avril 2019. URL : http://journals.openedition.org/lidil/4206 ;

DOI : 10.4000/lidil.4206

Ce document a été généré automatiquement le 19 avril 2019

(C) Lidil 


\section{Phrases complexes et maturité syntaxique : une comparaison entre des écrits d'élèves de 13 et 16 ans}

Complex Sentences and Syntactic Maturity: A Comparison between 8th and 11th Grade Students

Marie-Claude Boivin, Katrine Roussel et Reine Pinsonneault

\section{Problématique}

1 La recherche présentée dans cet article ${ }^{1}$ constitue une suite directe du travail de Boivin et Pinsonneault $(2014,2017)$ sur les erreurs linguistiques dans les textes des élèves québécois. Ce travail s'inscrit dans un contexte plus large de description de la maitrise de la norme écrite chez les élèves francophones et de réflexion sur ce thème (cf. Diepe, 1995 ; Lemonnier \& Gagnon, 2010 ; Manesse \& Cogis, 2007 ; MELS, 2010 ; Paret, 1988, 1991 ; Roy, Lafontaine \& Legros, 1995).

2 Le travail de Boivin et Pinsonneault $(2014,2017)$ se fonde sur un corpus de près de 1000 textes d'élèves rédigés dans un contexte d'épreuves d'écriture de fin d'année. Les résultats indiquent notamment que, à tous les niveaux scolaires, les erreurs de syntaxe sont plus nombreuses que les erreurs d'orthographe grammaticale. Ainsi, le nombre moyen d'erreurs de syntaxe par 100 mots (NM100) est de 6,31 en $2^{\mathrm{e}}$ secondaire (13 ans) et de 3,87 en $5^{\text {e }}$ secondaire $(16 \mathrm{ans})^{2}$, alors que le NM100 en orthographe grammaticale est de 3,34 et de 1,58 respectivement pour ces deux niveaux scolaires. Ces résultats montrent donc l'intérêt de se pencher sur les erreurs de syntaxe des élèves.

Un des résultats de cette étude qui semble demander un approfondissement concerne le NM100 lié à la construction des phrases complexes. En effet, la différence de NM100 pour les phrases complexes entre les élèves de $2^{\mathrm{e}}$ secondaire $(0,79)$ et ceux de $5^{\mathrm{e}}$ secondaire $(0,60)$, bien que significative $(p<0,005)$, est minime. Les élèves de $5^{\mathrm{e}}$ secondaire se trouvent donc à faire en moyenne 0,19 erreur de phrases complexes de moins par 
100 mots que ceux de $2^{\mathrm{e}}$ secondaire. Les erreurs de phrases complexes comprennent des erreurs relatives aux sous-catégories suivantes ${ }^{3}$ : coordination et juxtaposition; subordonnée corrélative ; subordonnée infinitive ou participiale ; subordonnée relative et choix du subordonnant; enchainement erroné de trois phrases ou plus; insertion d'incises; concordance des temps ${ }^{4}$; choix du mode dans les subordonnées. La faible amélioration observée entre 13 et 16 ans est d'autant plus étonnante que la maitrise de la phrase complexe est vue comme un indicateur de maturité syntaxique (Hunt, 1965 ; Paret, 1991).

4 La mesure utilisée par Boivin et Pinsonneault (2014, 2017), soit le NM100, rapporte le nombre d'erreurs au nombre de mots dans le texte. Le NM100 est utile, car il permet une comparaison du nombre d'erreurs entre des textes de longueurs variées et n'exige pas d'analyse du contenu des textes. Il serait toutefois pertinent de mettre le nombre d'erreurs en relation avec le nombre de contextes où l'erreur est possible dans le texte, plutôt qu'avec le nombre de mots. Par exemple, un élève qui n'a fait qu'une erreur de construction de phrase complexe dans un texte de 300 mots aurait un taux de 0,33 erreur par 100 mots $(0,33 \%)$, mais si le texte contient quatre phrases complexes, le taux d'erreur par contexte serait de 1 erreur pour 4 contextes $(25 \%)$. On peut donc s'interroger sur l'adéquation de la mesure donnée par le NM100; la mesure du nombre d'erreurs par contextes pourrait en révéler davantage sur la capacité des élèves à construire des phrases complexes. Cette mesure demande d'effectuer une analyse approfondie de ce que Boivin et Pinsonneault $(2014,2017)$ appellent la « composition syntaxique » des textes.

L'objectif général de cet article est donc d'explorer la différence entre les élèves de $2^{\mathrm{e}}$ secondaire et ceux de $5^{\mathrm{e}}$ secondaire en ce qui concerne la composition syntaxique de leurs textes ainsi que leur maitrise des phrases complexes. Cette exploration permettra de mieux cerner les paramètres de la maturité syntaxique chez les élèves, et par le fait même, de mieux cibler les difficultés des apprentis scripteurs et leurs sources en ce qui concerne la construction de phrases complexes.

6 L'article est organisé comme suit. La deuxième section présente le cadre conceptuel sousjacent à notre travail et indique les objectifs spécifiques de la recherche. La troisième section détaille la méthodologie employée pour l'analyse des textes. La quatrième section fait état des résultats obtenus et la cinquième en présente une discussion. La conclusion propose des pistes pour la recherche future.

\section{Cadre conceptuel}

7 Notre travail s'inscrit dans le cadre théorique de la grammaire nouvelle ou moderne (cf. Boivin \& Pinsonneault, 2008 ; Chartrand, 1996 ; Gobbe, 1980 ; Nadeau \& Fisher, 2006). Dans cette section, nous ferons d'abord état de quelques définitions liées à la notion de phrase qui serviront à l'analyse de la composition syntaxique des textes; nous présenterons ensuite le concept de maturité syntaxique.

\subsection{La notion de phrase complexe}

8 Nous adoptons une définition syntaxique de la phrase $\mathrm{P}$, soit la combinaison de deux groupes syntaxiques obligatoires $\mathrm{GN}$ et GV, et d'un ou plusieurs groupes syntaxiques facultatifs (cf. Riegel, Pellat \& Rioul, 2016). Nous utilisons la notion de phrase graphique 
uniquement pour délimiter, de la majuscule au point, les phrases dans les textes des élèves.

Les phrases, que ce soit du point de vue graphique ou syntaxique (Riegel, Pellat \& Rioul, 2016), se scindent en deux grandes classes principales : les phrases simples et les phrases complexes. Une phrase simple est une phrase qui se limite à une seule phrase syntaxique, sans contenir de phrase subordonnée. La phrase complexe en (1) est composée de deux phrases simples, comme illustré en (2).

(1) L'automne, qui arrive bientôt, nous offrira de beaux paysages.

(2) a. L'automne nous offrira de beaux paysages.

b. L'automne arrive bientôt.

10 Il existe trois procédés de formation des phrases complexes: la juxtaposition, la coordination et la subordination (Boivin \& Pinsonneault, 2008; Riegel, Pellat \& Rioul, 2016). L'utilisation de ces procédés, seuls ou en combinaison, détermine la structure syntaxique des phrases complexes.

11 La juxtaposition consiste à disposer deux ou plusieurs phrases syntaxiques côte à côte, séparées à l'écrit par une virgule, un point-virgule ou un deux-points. Les deux phrases ainsi juxtaposées sont toutes deux des phrases autonomes sur le plan syntaxique. La phrase en (3) en est un exemple.

(3) Je partirai loin, je découvrirai le monde.

12 La coordination fonctionne comme la juxtaposition, mais elle joint deux phrases syntaxiques à l'aide d'un coordonnant (par exemple, mais, ou, et, ni, or et car), comme en (4).

(4) Je partirai loin et je découvrirai le monde.

13 Il en va autrement pour la subordination, qui consiste à enchâsser une phrase à l'intérieur d'une phrase syntaxique ou d'un groupe syntaxique, souvent au moyen d'un subordonnant (que, qui, dont, parce que, si, etc.). La phrase subordonnée, contrairement aux phrases juxtaposées et coordonnées, n'est pas autonome sur le plan syntaxique.

Les subordonnées sont généralement classées selon leur construction syntaxique, soit les subordonnées relatives (5), complétives (6), corrélatives (7), infinitives (8), (9) et (10), participiales (11) et (12), ou selon leur fonction grammaticale, soit les subordonnées compléments de phrase (13) et adjointes (14). Elles sont délimitées par des crochets dans les exemples ci-dessous.

(5) L'automne, [qui arrive bientôt], nous offrira de beaux paysages.

(6) Lily pense [que nous sommes trop bruyants].

(7) Elle était tellement ravie par cette proposition [qu'elle l'a acceptée sur-le-champ].

(8) Jade connait un bel endroit [où dormir].

(9) Elle pense [aller ailleurs].

(10) [Planifier un voyage] me semble terriblement compliqué.

(11) Les parents ont bien suivi les consignes [figurant sur le feuillet d'information].

(12) [En parlant ainsi], elle en a offusqué quelques-uns.

(13) Ils ont nettoyé la plage [pendant que nous nous occupions de la propreté du lac].

(14) Je vais te croire, puisque tu me le répètes sans cesse.

Ainsi, une phrase syntaxique peut être simple ou complexe, et si elle est complexe, elle sera construite à partir des procédés de juxtaposition, de coordination, de subordination, en combinaison ou non. On peut aussi former une phrase complexe par insertion de phrases, incises ou incidentes (cf. Ce problème, c'est bien connu, n'a pas été résolu), mais elles ne font pas l'objet d'une étude approfondie dans cet article. 


\subsection{La maturité syntaxique} l'ensemble des caractéristiques syntaxiques «relevées dans l'écrit des élèves les plus avancés dans leur scolarité » (Paret, 1991). Différents critères permettent de caractériser la maturité syntaxique des élèves. La mobilité des constituants à l'intérieur de la phrase, c'est-à-dire le fait de produire des phrases dans lesquelles les constituants mobiles sont déplacés en dehors de leur position canonique, est un indice de maturité syntaxique. Un exemple en est donné en (15), où le groupe prépositionnel en ce beau matin d'automne, complément de phrase, est déplacé entre le GN sujet et le GV prédicat.

(15) Le soleil, en ce beau matin d'automne, illuminait d'un éclat radieux les feuilles $d u$ pommier.

Un deuxième indice de maturité syntaxique est donné par le nombre de constituants dans une phrase et dans les groupes. Ainsi, le fait d'avoir plusieurs compléments de phrase dans la phrase ou d'avoir plusieurs compléments du nom dans un GN, comme en (16), traduit une plus grande maturité syntaxique.

(16) Le soleil, dans la vallée, en ce beau matin d'automne, illuminait d'un éclat radieux les feuilles du pommier, qui bruissaient au vent.

Un troisième critère de maturité syntaxique, très saillant, est la proportion importante de phrases complexes. Selon Paret (1988), la maturation syntaxique permet aux élèves « un élargissement de leur champ de possibilités syntaxiques leur offrant [notamment] [...] une plus grande maîtrise de la phrase complexe» (p. 201). Cette maturation ferait donc en sorte que les élèves s'approprient de plus en plus les procédés de formation de phrases complexes, en particulier la subordination, parce qu'ils arrivent ainsi à condenser «davantage d'informations en un seul énoncé » (Paret, 1988, p. 185). Une plus grande maturité syntaxique étant atteinte par les élèves les plus avancés dans leur cheminement (Hunt, 1965 ; Paret, 1991), les caractéristiques syntaxiques des productions écrites de ces élèves deviennent donc un point de comparaison avec lequel peuvent être mis en relation les textes d'élèves plus jeunes ou plus faibles.

La question de la maturité syntaxique est pertinente pour notre travail puisqu'elle offre, par la prise en compte des contextes syntaxiques et de leurs caractéristiques, une entrée complémentaire pour décrire les compétences syntaxiques des élèves du secondaire. Nous souhaitons tenir compte de la composition syntaxique des textes pour explorer la différence entre les compétences en construction de phrases complexes d'élèves de niveaux scolaires différents ( $2^{\mathrm{e}}$ et $5^{\mathrm{e}}$ secondaire) et de classements différents (forts et faibles).

Afin d'atteindre notre objectif général qui est d'explorer la différence entre la maitrise des phrases complexes des élèves de $2^{\mathrm{e}}$ et $5^{\mathrm{e}}$ secondaire, nous viserons les objectifs spécifiques suivants :

1. Décrire et comparer la composition syntaxique des textes d'élèves relativement aux phrases complexes, en fonction à la fois de l'âge et du classement des élèves ;

2. Comparer la maitrise des phrases complexes des élèves selon leur âge et leur classement à l'aide d'une nouvelle mesure mettant en relation le nombre d'erreurs et le nombre de contextes où l'erreur est possible (le nombre d'erreurs par contextes). 


\section{Méthodologie}

21 Comme notre travail se veut une suite au travail amorcé par Boivin et Pinsonneault (2014, 2017), nous décrirons tout d'abord brièvement leur échantillon, dont le nôtre est issu. Nous détaillerons ensuite les codes utilisés pour l'analyse de la composition syntaxique des textes.

Le corpus de Boivin et Pinsonneault $(2014,2017)$ comporte 969 textes d'élèves, dont 250 textes d'élèves de $4^{\mathrm{e}}$ année du primaire ( 9 ans), 220 de $6^{\mathrm{e}}$ année du primaire (11 ans), 250 de $2^{\mathrm{e}}$ année du secondaire (13 ans) et 249 de $5^{\mathrm{e}}$ année du secondaire ( 16 ans). Il a été constitué à partir d'un vaste ensemble d'épreuves d'écriture fournies par le ministère de l'Éducation, du Loisir et du Sport du Québec (MELS) ${ }^{5}$. Ces textes ont été sélectionnés à partir d'un plan d'échantillonnage aléatoire stratifié selon le sexe, tout en respectant les proportions de fréquentation des écoles des secteurs public et privé. Cet échantillon est donc représentatif de la population d'élèves pour chaque niveau scolaire mais ne nous permet pas de prendre en compte des variables socioéconomiques.

Afin de répondre à notre premier objectif spécifique, soit de décrire et de comparer la composition syntaxique des textes relativement aux phrases complexes, nous devons identifier dans les textes les différents contextes de phrases complexes. Nous avons choisi d'effectuer ce travail sur un échantillon limité de textes représentatifs d'élèves forts et faibles pour chaque niveau. Ce choix permet de distinguer dans notre échantillon deux types d'élèves "avancés » sur le plan de la maturité syntaxique, soit les plus âgés et les plus forts pour chaque niveau. Ceci permet une double mise en contraste pour la réflexion sur la maturité syntaxique ${ }^{6}$.

Pour constituer notre échantillon, nous avons d'abord ordonné tous les textes d'élèves du niveau secondaire du corpus de Boivin et Pinsonneault $(2014,2017)$ selon leur nombre total d'erreurs par 100 mots (N100), toutes catégories d'erreurs confondues (syntaxe, orthographe grammaticale et orthographe lexicale). Ce classement nous a permis de diviser les élèves en cinq tranches de 50 élèves selon leur position. De manière à exclure les cas extrêmes, nous avons ensuite subdivisé les tranches d'élèves les plus forts et les plus faibles en deux sous-groupes de 25, pour obtenir sept tranches (voir tableau 1). Les 250 élèves de $2^{\mathrm{e}}$ secondaire et les 249 élèves de $5^{\mathrm{e}}$ secondaire sont donc classés selon les catégories suivantes : très fort, fort, moyen-fort, moyen, moyen-faible, faible et très faible. Le nombre d'élèves $(N)$, le nombre moyen d'erreurs par 100 mots (NM100), les nombres d'erreurs par 100 mots minima et maxima (N100 min et N100 max) de même que l'écart-type ont été calculés pour chacune de ces tranches.

Tableau 1. - Classement des élèves de l'échantillon.

\begin{tabular}{|c|c|c|c|c|c|c|c|}
\hline \multicolumn{7}{|c|}{$2^{\mathrm{e}}$ année du secondaire $(N=250 ; \mathrm{NM} 100: 13,35)$} \\
\hline & très fort & fort & moyen-fort & moyen & moyen-faible & faible & très faible \\
\hline$N$ & 25 & 25 & 50 & 50 & 50 & 25 & 25 \\
\hline NM100 & 3,4 & 5,9 & 8,5 & 11,8 & 16,3 & 21,5 & 28,7 \\
\hline
\end{tabular}




\begin{tabular}{|c|c|c|c|c|c|c|c|c|}
\hline N100 min. & 0,5 & 4,9 & 6,9 & 10,1 & 13,7 & 19,5 & 24,0 \\
\hline N100 max. & 4,8 & 6,9 & 9,9 & 13,6 & 19,4 & 23,7 & 47,3 \\
\hline Écart-type & 1,2 & 0,1 & 0,9 & 1,1 & 1,8 & 1,2 & 5,1 \\
\hline \multicolumn{2}{|c|}{$\mathbf{5}^{\mathbf{e}}$ année du secondaire $(\mathrm{N}=249 ; \mathrm{NM} 100: 6,99)$} & & \\
\hline & très fort & fort & moyen-fort & moyen & moyen-faible & faible & très faible \\
\hline N & 25 & 25 & 50 & 50 & 50 & 25 & 24 \\
\hline NM100 & 2,2 & 3,1 & 4,4 & 6,2 & 8,5 & 10,6 & 16,2 \\
\hline N100 min. & 1,3 & 2,7 & 3,6 & 5,3 & 7,3 & 9,7 & 12,1 \\
\hline N100 max. & 2,7 & 3,6 & 5,2 & 7,3 & 9,7 & 12,0 & 22,5 \\
\hline Écart-type & 0,4 & 0,2 & 0,5 & 0,6 & 0,7 & 0,6 & 2,4 \\
\hline
\end{tabular}

Nous avons ensuite sélectionné cinq textes d'élèves forts et cinq textes d'élèves faibles dans chaque groupe d'âge, pour un total de 20 textes. Le choix de textes forts et faibles permet de faire ressortir un contraste sans aller dans des cas extrêmes, qui seraient peutêtre moins représentatifs des forces et faiblesses des élèves. Le choix de l'âge est lié au corpus de textes utilisés par Boivin et Pinsonneault (2014, 2017), qui contient des épreuves ministérielles d'écriture ayant lieu à la fin de la $2^{\mathrm{e}}$ et $5^{\mathrm{e}}$ année du secondaire. Ces deux niveaux permettent d'établir un contraste intéressant pour évaluer la maturité syntaxique. La sélection de ces textes s'est effectuée de façon aléatoire en veillant à respecter les proportions obtenues par Boivin et Pinsonneault $(2014,2017)$ pour le sexe des élèves et leur secteur (privé ou public). Les données sociodémographiques de notre échantillon (voir tableau 2) indiquent que les nombres de filles et de garçons sont égaux (10 filles et 10 garçons), tout comme le nombre d'élèves de chaque niveau scolaire (10 de 2 ${ }^{\mathrm{e}}$ secondaire et $10 \mathrm{de} 5^{\mathrm{e}}$ secondaire) et de chaque tranche retenue ( 10 forts et 10 faibles). Enfin, on remarque qu'il y a quatre fois plus d'élèves appartenant au secteur public que d'élèves du secteur privé ( 16 du secteur public et 4 du secteur privé), tout comme dans l'échantillon initial.

Tableau 2. - Description de l'échantillon $(N=20)$.

\begin{tabular}{|c|c|c|c|c|c|c|c|}
\hline \multicolumn{2}{|c|}{} & Privé & Public & Total & Fort & Faible & Total \\
\hline \multirow{2}{*}{$2^{\text {e }}$ sec. } & M & 1 & 4 & 5 & 3 & 2 & 5 \\
\cline { 2 - 8 } & F & 1 & 4 & 5 & 2 & 3 & 5 \\
\hline 5 sec. & M & 1 & 4 & 5 & 2 & 3 & 5 \\
\cline { 2 - 8 } & F & 1 & 4 & 5 & 3 & 2 & 5 \\
\hline Total & 4 & 16 & $\mathbf{2 0}$ & 10 & 10 & $\mathbf{2 0}$ \\
\hline
\end{tabular}


Nous avons identifié notre échantillon $(N=20)$ sur une échelle ordinale (voir fig. 1$)$ afin de mieux le situer par rapport à celui de Boivin et Pinsonneault $(2014,2017)(N=499)$, les textes retenus étant représentés par les 20 points.

Figure 1. - Situation de l'échantillon sur une échelle ordinale.

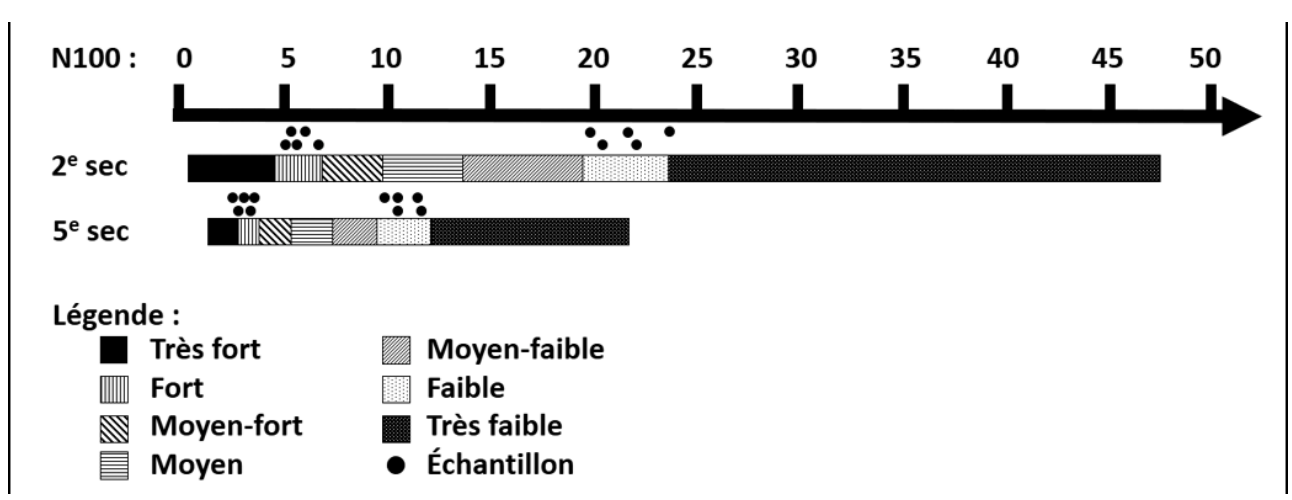

Voici une brève description des performances générales pour les vingt élèves de l'échantillon. Pour les cinq forts de $2^{\mathrm{e}}$ secondaire, le N100 se situe entre 4,9 et 6,9 , alors qu'il se situe entre 2,7 et 3,6 chez les forts de $5^{\mathrm{e}}$ secondaire. Chez les faibles, il varie entre 19,5 et 23,7 chez les élèves de $2^{\mathrm{e}}$ secondaire, et entre 9,7 et 12,0 chez ceux de $5^{\mathrm{e}}$ secondaire. On remarque qu'entre les forts et les faibles de chaque âge, les performances sont presque quatre fois meilleures chez les forts. De plus, la performance générale des forts de $2^{\mathrm{e}}$ secondaire est meilleure que celle des faibles de $5^{\mathrm{e}}$ secondaire et se situe de surcroit plus près de celle des forts de $5^{\mathrm{e}}$ secondaire.

Une fois notre échantillon formé, nous avons importé les textes dans le logiciel d'analyse qualitative HyperRESEARCH en vue d'en analyser la composition syntaxique. Un codage a permis de dénombrer les phrases graphiques, les phrases simples, les phrases complexes, les phrases juxtaposées ou coordonnées, les subordonnées relatives, les subordonnées corrélatives, les subordonnées participiales ou infinitives et tout autre type de subordonnées contenues dans chacun des textes. Les subordonnées « autres » regroupent les subordonnées compléments de phrase et les adjointes, les subordonnées complétives tensées, les incises et les incidentes. Ces subordonnées se retrouvent dans la catégorie " autres" soit parce qu'elles présentaient peu d'erreurs ou peu d'occurrences dans le corpus, et non en raison de caractéristiques linguistiques communes. Ce classement a été motivé par la façon dont les erreurs ont été originalement codées dans le travail de Boivin et Pinsonneault $(2014,2017)$. Bien que ce classement présente des limites, notons que les études antérieures ayant porté sur le classement des erreurs, soit ne s'intéressaient pas à la syntaxe (cf. Manesse \& Cogis, 2007), soit ne caractérisaient pas aussi finement ces erreurs, se limitant à une catégorie "syntaxe» (Lefrançois et coll., 2008; Roy, Lafontaine \& Legros, 1995) ou à une catégorie "subordination» (Libersan, 2003). La liste des codes utilisés ainsi que des exemples ${ }^{7}$ tirés de notre corpus se trouvent au tableau 3.

Tableau 3. - Liste des codes et exemples.

\begin{tabular}{|l|l}
\hline Code & Exemples tirés de notre corpus \\
\hline
\end{tabular}




\begin{tabular}{|c|c|}
\hline Phrase graphique & $\begin{array}{l}\text { Le voyage allait de bon train, jusqu'à la nuit du } 29 \text { mai } 1914 . \\
\text { Survenu à peine deux ans après la tragédie du Titanic, la catastrophe de } \\
\text { l'Empress of Ireland détient, encore aujourd'hui, le reccord de victimes en } \\
\text { temps de paix. }\end{array}$ \\
\hline Phrase simple & À 1 heure 30 minutes, le pilote débarque à l'approche de Pointe-au-père. \\
\hline Phrase complexe & $\begin{array}{l}\text { Tout d'abord, l'Empress était un transatlantique qui possédait } 40 \text { chaloupes } \\
\text { qui pouvaient accueillir un total de } 1850 \text { personnes pour } 1477 \text { passagers et } \\
\text { membres d'équipage. }\end{array}$ \\
\hline $\begin{array}{l}\text { Phrases juxtaposées / } \\
\text { coordonnées }\end{array}$ & $\begin{array}{l}\text { Il était trop tard, le « Storstad » était trop très [...]. } \\
\text { Finalement, je crois qu'il est important de connaître cette tragédie car elle a } \\
\text { été en partie étouffé par le début de la guerre mondiale. }\end{array}$ \\
\hline $\begin{array}{l}\text { Subordonnée } \\
\text { corrélative }\end{array}$ & $\begin{array}{l}\text { La douleur et la mort qu'a subi ces gens étaient si fortes qu'on se souvient } \\
\text { encore des châtiments qu'elle exercait sur les malades. }\end{array}$ \\
\hline Subordonnée relative & $\begin{array}{l}\text { Le commandant apperçoit facilement les feux de position d'un charbonnier } \\
\text { qui arrive en direction opposé. }\end{array}$ \\
\hline $\begin{array}{l}\text { Subordonnée } \\
\text { infinitive / participiale }\end{array}$ & $\begin{array}{l}\text { Finalement, je crois qu'il est important de connaître cette tragédie [...]. } \\
\text { Perdant de vue les feux à travers le brouillard, le commandant fait } \\
\text { stopper les moteurs. }\end{array}$ \\
\hline Subordonnées autres & $\begin{array}{l}\text { Même si cette tragédie a fait plusieurs centaines de victimes, elle a } \\
\text { apporté quelques points positifs. } \\
\text { Aussi, des embarcations seront installés sur le pont supérieur, pour qu'elles } \\
\text { flottent instantanément durant un naufrage. } \\
\text { Finalement, je crois qu'il est important de connaître cette tragédie [...]. } \\
410 \text { étudiants du collège Victoriaville, } 400 \text { serons touchés par cette maladie. “ } \\
\text { Dit par Magda Fahrni, historienne et spécialiste de la grippe espagnole. }\end{array}$ \\
\hline
\end{tabular}

Une phrase complexe, comme celle en (17), tirée d'un texte d'élève fort de $2^{\mathrm{e}}$ secondaire, peut recevoir plus d'un code.

(17) Tout le monde se rappelle de la catastrophe [qui a frappé un des navires précédents le "Titanic »], [mais cela n'empêche personne [d'être de bonne humeur] [et [de fêter]]].

30 En effet, il s'agit d'une phrase qui reçoit les codes phrase graphique, phrase complexe, subordonnée relative, coordination et subordonnée infinitive. Plusieurs combinatoires entre les codes utilisés pour la composition syntaxique sont donc possibles.

31 Les phrases graphiques sont déterminées à partir de la ponctuation et des indices typographiques utilisés par l'élève. Nous n'avons pas dénombré directement les phrases syntaxiques parce que nos calculs sont effectués en regard des phrases graphiques ${ }^{8}$.

32 Les données issues du codage ont permis de déterminer le nombre de contextes liés aux phrases complexes (composition syntaxique) et de calculer les taux d'erreurs liées aux phrases complexes selon le nombre de contextes possibles. Il s'agit d'un travail descriptif : le caractère significatif des différences n'est pas vérifié par des tests statistiques. Précisons que pour le codage des erreurs, les textes ont été codés par quatre codeuses et 
qu'un contrecodage a été effectué sur $6 \%$ des textes du corpus. La cohérence interne du codage des erreurs syntaxiques entre les codeuses, calculé à l'aide du coefficient de corrélation intraclasse, est de 0,871 , ce qui est excellent (le maximum étant 1 ). En ce qui concerne la composition syntaxique des 20 textes, le codage a été effectué par une seule codeuse.

\section{Résultats}

Dans cette section, nous présenterons les principaux résultats obtenus suite au codage des 20 textes de notre corpus. Nous commencerons par présenter la description de la composition syntaxique des textes retenus (objectif 1 ) et poursuivrons avec les résultats relatifs aux pourcentages de contextes de phrases complexes contenant des erreurs (objectif 2).

\subsection{Composition syntaxique}

Nous présenterons la distribution des phrases simples et complexes dans les phrases graphiques de chacun des textes de notre échantillon afin d'en décrire la composition syntaxique. Nous indiquons d'abord le nombre d'occurrences de chaque type de phrases dans chacun des 20 textes retenus (voir tableau 4). À titre indicatif, le nombre de mots figure également dans ce tableau.

Tableau 4. - Composition syntaxique des phrases graphiques.

\begin{tabular}{|c|c|c|c|c|c|c|c|}
\hline \multirow{2}{*}{ niv. } & \multirow{2}{*}{ cas } & \multirow{2}{*}{$N$ mots } & \multirow{2}{*}{$N$ phrases graphiques } & \multicolumn{2}{|c|}{$\begin{array}{c}N \text { phrases } \\
\text { simples }\end{array}$} & \multicolumn{2}{|c|}{$\begin{array}{l}N \text { phrases } \\
\text { complexes }\end{array}$} \\
\hline & & & & $\mathrm{N}$ & $\%$ & $\mathrm{~N}$ & $\%$ \\
\hline \multirow{6}{*}{$\begin{array}{l}2^{\mathrm{e}} \mathrm{sec} . \\
\text { forts }\end{array}$} & 1 & 383 & 25 & 12 & $48 \%$ & 13 & $52 \%$ \\
\hline & 2 & 430 & 25 & 9 & $36 \%$ & 16 & $64 \%$ \\
\hline & 3 & 333 & 26 & 14 & $54 \%$ & 12 & $46 \%$ \\
\hline & 4 & 342 & 17 & 4 & $23 \%$ & 13 & $77 \%$ \\
\hline & 5 & 329 & 21 & 10 & $48 \%$ & 11 & $52 \%$ \\
\hline & moyenne & 363,4 & 23 & 10 & $43 \%$ & 13 & $57 \%$ \\
\hline \multirow{5}{*}{$\begin{array}{l}2^{\mathrm{e}} \mathrm{sec} . \\
\text { faibles }\end{array}$} & 6 & 301 & 19 & 7 & $37 \%$ & 12 & $63 \%$ \\
\hline & 7 & 448 & 25 & 8 & $32 \%$ & 17 & $68 \%$ \\
\hline & 8 & 410 & 30 & 14 & $47 \%$ & 16 & $53 \%$ \\
\hline & 9 & 422 & 25 & 9 & $36 \%$ & 16 & $64 \%$ \\
\hline & 10 & 334 & 21 & 9 & $43 \%$ & 12 & $57 \%$ \\
\hline
\end{tabular}




\begin{tabular}{|c|c|c|c|c|c|c|c|}
\hline & moyenne & 383 & 24 & 9 & $38 \%$ & 15 & $62 \%$ \\
\hline \multirow{6}{*}{$\begin{array}{l}5^{\mathrm{e}} \mathrm{sec} \\
\text { forts }\end{array}$} & 11 & 478 & 26 & 5 & $19 \%$ & 21 & $81 \%$ \\
\hline & 12 & 832 & 48 & 14 & $29 \%$ & 34 & $71 \%$ \\
\hline & 13 & 779 & 40 & 4 & $10 \%$ & 36 & $90 \%$ \\
\hline & 14 & 550 & 17 & 2 & $12 \%$ & 15 & $88 \%$ \\
\hline & 15 & 610 & 34 & 8 & $24 \%$ & 26 & $76 \%$ \\
\hline & moyenne & 649,8 & 33 & 7 & $21 \%$ & 26 & $79 \%$ \\
\hline \multirow{6}{*}{$\begin{array}{c}5^{\mathrm{e}} \text { sec. } \\
\text { faibles }\end{array}$} & 16 & 540 & 23 & 2 & $9 \%$ & 21 & $91 \%$ \\
\hline & 17 & 559 & 28 & 5 & $18 \%$ & 23 & $82 \%$ \\
\hline & 18 & 535 & 31 & 5 & $16 \%$ & 26 & $84 \%$ \\
\hline & 19 & 498 & 30 & 10 & $33 \%$ & 20 & $67 \%$ \\
\hline & 20 & 531 & 13 & 1 & $8 \%$ & 12 & $92 \%$ \\
\hline & moyenne & 532,6 & 25 & 5 & $20 \%$ & 20 & $80 \%$ \\
\hline
\end{tabular}

Le nombre moyen de phrases graphiques des élèves de $2^{\mathrm{e}}$ secondaire, forts ou faibles, est de 23 ou 24 . Chez les élèves de $5^{\mathrm{e}}$ secondaire, les faibles produisent 25 phrases graphiques, alors que les forts en produisent 33. On constate donc que les élèves faibles de $5^{\mathrm{e}}$ secondaire font un nombre de phrases graphiques comparable à celui des élèves de $2^{\mathrm{e}}$ secondaire, tout en produisant des textes plus longs (en moyenne 532 mots comparativement à 383). Leurs phrases sont plus longues et ils produisent plus de phrases complexes. Sept élèves de $5^{\mathrm{e}}$ secondaire sur 10 ont produit un nombre très faible de phrases simples ( 5 ou moins), alors qu'un seul élève de $2^{\mathrm{e}}$ secondaire est dans cette situation. Ces sept élèves comprennent trois forts et quatre faibles.

À l'intérieur de chacun des niveaux scolaires, on observe peu de différence entre les forts et les faibles dans la proportion de phrases simples et complexes. Les textes des élèves de $2^{\mathrm{e}}$ secondaire contiennent environ $40 \%$ de phrases simples et $60 \%$ de phrases complexes, tandis que ceux des élèves de $5^{\mathrm{e}}$ secondaire renferment environ $20 \%$ de phrases simples et $80 \%$ de phrases complexes. Ainsi, on remarque une augmentation d'environ 20 points de la proportion de phrases complexes entre 13 et 16 ans.

Nous présentons maintenant la composition syntaxique des phrases complexes. Nous avons relevé le nombre de contextes de phrases complexes ${ }^{9}$ et les avons classés en deux grands types: phrases juxtaposées ou coordonnées et phrases subordonnées (voir tableau 5). 
Tableau 5. - Composition syntaxique des phrases complexes.

\begin{tabular}{|c|c|c|c|c|c|c|}
\hline \multirow{2}{*}{ niv. } & \multirow{2}{*}{ cas } & \multirow{2}{*}{$\begin{array}{l}N \text { contextes phrases } \\
\text { complexes }\end{array}$} & \multicolumn{2}{|c|}{$\begin{array}{l}\text { P juxtaposées / } \\
\text { coordonnées }\end{array}$} & \multicolumn{2}{|c|}{ P subordonnées } \\
\hline & & & $N$ & $\%$ & $N$ & $\%$ \\
\hline \multirow{6}{*}{$\begin{array}{l}2^{\mathrm{e}} \mathrm{sec} \\
\text { forts }\end{array}$} & 1 & 23 & 2 & $9 \%$ & 21 & $91 \%$ \\
\hline & 2 & 37 & 10 & $27 \%$ & 27 & $73 \%$ \\
\hline & 3 & 19 & 3 & $16 \%$ & 16 & $84 \%$ \\
\hline & 4 & 28 & 9 & $32 \%$ & 19 & $68 \%$ \\
\hline & 5 & 18 & 4 & $22 \%$ & 14 & $78 \%$ \\
\hline & moyenne & 25 & 6 & $21 \%$ & 19 & $79 \%$ \\
\hline \multirow{6}{*}{$\begin{array}{l}2^{\mathrm{e}} \mathrm{sec} . \\
\text { faibles }\end{array}$} & 6 & 25 & 6 & $24 \%$ & 19 & $76 \%$ \\
\hline & 7 & 39 & 14 & $36 \%$ & 25 & $64 \%$ \\
\hline & 8 & 22 & 7 & $32 \%$ & 15 & $68 \%$ \\
\hline & 9 & 34 & 15 & $44 \%$ & 19 & $56 \%$ \\
\hline & 10 & 25 & 10 & $40 \%$ & 15 & $60 \%$ \\
\hline & moyenne & 29 & 10 & $35 \%$ & 19 & $65 \%$ \\
\hline \multirow{6}{*}{$\begin{array}{l}5^{\mathrm{e}} \mathrm{sec} . \\
\text { forts }\end{array}$} & 11 & 52 & 13 & $25 \%$ & 39 & $75 \%$ \\
\hline & 12 & 90 & 16 & $18 \%$ & 74 & $82 \%$ \\
\hline & 13 & 102 & 15 & $15 \%$ & 87 & $85 \%$ \\
\hline & 14 & 52 & 5 & $10 \%$ & 47 & $90 \%$ \\
\hline & 15 & 75 & 20 & $27 \%$ & 55 & $73 \%$ \\
\hline & moyenne & 74 & 14 & $19 \%$ & 60 & $81 \%$ \\
\hline \multirow{5}{*}{$\begin{array}{l}5^{\mathrm{e}} \mathrm{sec} . \\
\text { faibles }\end{array}$} & 16 & 62 & 21 & $34 \%$ & 41 & $66 \%$ \\
\hline & 17 & 73 & 17 & $23 \%$ & 56 & $77 \%$ \\
\hline & 18 & 69 & 10 & $14 \%$ & 59 & $86 \%$ \\
\hline & 19 & 49 & 10 & $20 \%$ & 39 & $80 \%$ \\
\hline & 20 & 75 & 18 & $24 \%$ & 57 & $76 \%$ \\
\hline
\end{tabular}




\begin{tabular}{|l|l|l|l|l|l|}
\hline moyenne & 65 & 15 & $23 \%$ & 50 & $77 \%$ \\
\hline
\end{tabular}
proportion de phrases juxtaposées ou coordonnées diminue avec l'âge, plus particulièrement chez les faibles, alors que la proportion de phrases subordonnées augmente. Peu importe l'âge, la proportion de subordonnées dans les phrases complexes est plus élevée chez les forts que chez les faibles. Cette proportion est, en $2^{\mathrm{e}}$ secondaire, de $79 \%$ chez les forts et de $65 \%$ chez les faibles, et, en $5^{\text {e }}$ secondaire, de $81 \%$ chez les forts et de $77 \%$ chez les faibles. L'écart entre forts et faibles est moins marqué en $5^{\mathrm{e}}$ secondaire. On remarque aussi que chez les forts des deux âges, ces proportions sont très semblables ( $79 \%$ et $81 \%$ de subordonnées).

avons ensuite examiné la répartition des différents types de subordonnées dans les textes des élèves (voir tableau 6), de manière à déterminer la proportion de subordonnées corrélatives, relatives, infinitives et participiales, de même que celle des autres subordonnées (complétives, compléments de phrases, adjointes, incises, incidentes).

Tableau 6. - Composition syntaxique des phrases subordonnées.

\begin{tabular}{|c|c|c|c|c|c|c|c|c|c|c|}
\hline \multirow{2}{*}{ niv. } & \multirow{2}{*}{ cas } & \multirow{2}{*}{$\begin{array}{c}N \\
\text { subordonnées }\end{array}$} & \multicolumn{2}{|c|}{ corrélatives } & \multicolumn{2}{|c|}{ relatives } & \multicolumn{2}{|c|}{$\begin{array}{l}\text { infinitives / } \\
\text { participiales }\end{array}$} & \multicolumn{2}{|c|}{ autres } \\
\hline & & & $N$ & $\%$ & $N$ & $\%$ & $N$ & $\%$ & $N$ & $\%$ \\
\hline \multirow{6}{*}{$\begin{array}{l}2^{\mathrm{e}} \mathrm{sec} \\
\text { forts }\end{array}$} & 1 & 21 & 0 & $0 \%$ & 5 & $24 \%$ & 10 & $48 \%$ & 6 & $28 \%$ \\
\hline & 2 & 27 & 0 & $0 \%$ & 7 & $26 \%$ & 14 & $52 \%$ & 6 & $22 \%$ \\
\hline & 3 & 16 & 1 & $6 \%$ & 2 & $13 \%$ & 9 & $56 \%$ & 4 & $25 \%$ \\
\hline & 4 & 19 & 1 & $5 \%$ & 6 & $32 \%$ & 12 & $63 \%$ & 0 & $0 \%$ \\
\hline & 5 & 14 & 1 & $7 \%$ & 3 & $21 \%$ & 6 & $43 \%$ & 4 & $29 \%$ \\
\hline & moyenne & 19 & 1 & $5 \%$ & 4 & $21 \%$ & 10 & $53 \%$ & 4 & $21 \%$ \\
\hline \multirow{6}{*}{$\begin{array}{l}2^{\mathrm{e}} \mathrm{sec} . \\
\text { faibles }\end{array}$} & 6 & 19 & 0 & $0 \%$ & 4 & $21 \%$ & 11 & $58 \%$ & 4 & $21 \%$ \\
\hline & 7 & 25 & 0 & $0 \%$ & 6 & $24 \%$ & 14 & $56 \%$ & 5 & $20 \%$ \\
\hline & 8 & 15 & 3 & $20 \%$ & 3 & $20 \%$ & 8 & $53 \%$ & 1 & $7 \%$ \\
\hline & 9 & 19 & 3 & $16 \%$ & 6 & $32 \%$ & 9 & $47 \%$ & 1 & $5 \%$ \\
\hline & 10 & 15 & 0 & $0 \%$ & 1 & $7 \%$ & 10 & $66 \%$ & 4 & $27 \%$ \\
\hline & moyenne & 19 & 1 & $5 \%$ & 4 & $21 \%$ & 11 & $58 \%$ & 3 & $16 \%$ \\
\hline \multirow{2}{*}{$\begin{array}{l}5^{\mathrm{e}} \mathrm{sec} . \\
\text { forts }\end{array}$} & 11 & 39 & 2 & $5 \%$ & 6 & $15 \%$ & 18 & $46 \%$ & 13 & $33 \%$ \\
\hline & 12 & 74 & 2 & $3 \%$ & 6 & $8 \%$ & 37 & $50 \%$ & 29 & $39 \%$ \\
\hline
\end{tabular}




\begin{tabular}{|c|c|c|c|c|c|c|c|c|c|c|}
\hline & 13 & 87 & 1 & $1 \%$ & 12 & $14 \%$ & 54 & $62 \%$ & 20 & $23 \%$ \\
\hline & 14 & 47 & 5 & $11 \%$ & 13 & $28 \%$ & 15 & $32 \%$ & 14 & $30 \%$ \\
\hline & 15 & 55 & 0 & $0 \%$ & 9 & $16 \%$ & 31 & $56 \%$ & 15 & $27 \%$ \\
\hline & moyenne & 60 & 2 & $3 \%$ & 9 & $15 \%$ & 31 & $52 \%$ & 18 & $30 \%$ \\
\hline & 16 & 41 & 2 & $5 \%$ & 7 & $17 \%$ & 20 & $49 \%$ & 12 & $29 \%$ \\
\hline & 17 & 56 & 1 & $2 \%$ & 10 & $18 \%$ & 33 & $59 \%$ & 12 & $21 \%$ \\
\hline$r$ & 18 & 59 & 1 & $2 \%$ & 14 & $24 \%$ & 39 & $66 \%$ & 5 & $8 \%$ \\
\hline faibles & 19 & 39 & 1 & $3 \%$ & 4 & $10 \%$ & 25 & $64 \%$ & 9 & $23 \%$ \\
\hline & 20 & 57 & 0 & $0 \%$ & 14 & $24 \%$ & 30 & $53 \%$ & 13 & $23 \%$ \\
\hline & moyenne & 50 & 1 & $2 \%$ & 10 & $20 \%$ & 29 & $58 \%$ & 10 & $20 \%$ \\
\hline
\end{tabular}

Les subordonnées infinitives et participiales constituent dans tous les cas plus de la moitié des contextes de phrases complexes. Suivent les subordonnées relatives et les subordonnées "autres", dont les proportions sont similaires. Les subordonnées corrélatives sont les moins nombreuses.

De façon générale, la répartition des types de subordonnées est assez similaire entre les forts et les faibles d'une part et entre les élèves de 13 et 16 ans d'autre part. Il existe une différence entre les forts et les faibles aux deux niveaux scolaires: la proportion d'infinitives et de participiales est de 5 à 6 points plus élevée chez les faibles que chez les forts, et la proportion de subordonnées de catégorie « autres » est de 5 à 10 points plus élevée chez les forts que chez les faibles.

Chez les élèves de $5^{\mathrm{e}}$ secondaire, on observe un peu moins de subordonnées relatives du côté des forts $(15 \%)$ que des faibles $(20 \%)$, alors que les élèves de $2^{\mathrm{e}}$ secondaire, peu importe leur classement, produisent en moyenne $21 \%$ de subordonnées relatives. Chez les élèves forts de $5^{\mathrm{e}}$ secondaire, la proportion de subordonnées « autres » est de $30 \%$; tandis que chez les élèves faibles de $2^{\mathrm{e}}$ secondaire, ces subordonnées sont produites presque deux fois moins de fois ( $16 \%)$. De plus, les élèves forts de $5^{\mathrm{e}}$ secondaire produisent davantage de subordonnées " autres » que leurs pairs plus faibles $(20 \%)$ et que leurs pairs plus jeunes (21\%).

\subsection{Taux d'erreurs par contexte}

Rappelons notre deuxième objectif. À l'aide d'une nouvelle mesure mettant en relation le nombre d'erreurs et le nombre de contextes où l'erreur est possible, nous entendons comparer la maitrise des phrases complexes des élèves selon leur niveau scolaire et leur classement.

À titre indicatif, voici quelques exemples d'erreurs liées aux phrases complexes, généralement tirés des 20 textes du corpus, et les codes qui leur sont associés. 
Subordonnée relative et choix du subordonnant :

(18) Il a 40 chaloupe qu'il peuvent contenir 1850 personne.

Enchainement erroné de trois phrases ou plus :

(19) C'étais sûrment une traverssé comme une autre le temps étais beau le ciel étais clair et il y avait quelque passage de bume et tout semblaient si bien allé.

Subordonnée corrélative :

(20) Tellement qu'ils sont accour d'idée, ils mettent en vente du sirops pour le rhume et même des pilules pour soigner les nerfs ${ }^{10}$.

Choix du mode dans la subordonnée :

(21) Imaginez si les adultes n'auraient pas le droit de vote, mais que les jeunes oui.

Subordonnée infinitive ou participiale :

(22) Ne sachant plus quoi faire, la paralysi de toute vie sociale, est ordonné.

Coordination et juxtaposition :

(23) J'ai besoin de rencontrer et de discuter avec des gens pour améliorer mes compétences sociales.

Nous présentons dans le tableau 7, pour chacun des vingt textes de l'échantillon, le nombre d'erreurs de phrases complexes, le nombre de mots, le nombre de contextes de phrases complexes de même que le N100 lié à la construction des phrases complexes et le pourcentage de contextes de phrases complexes contenant une erreur. Contrairement aux tableaux précédents, ce tableau ordonne les cas selon le pourcentage d'erreurs par contexte.

Tableau 7. - Performances liées aux phrases complexes.

\begin{tabular}{|c|c|c|c|c|c|c|}
\hline niv. & cas & $\begin{array}{c}N \text { erreurs } \\
\text { P complexes }\end{array}$ & $N$ mots & $\begin{array}{l}N \text { contextes } \\
\text { P complexes }\end{array}$ & $\begin{array}{c}N 100 \\
\text { P complexes }\end{array}$ & $\%$ contextes avec erreur \\
\hline \multirow{6}{*}{$\begin{array}{l}2^{\mathrm{e}} \mathrm{sec} . \\
\text { forts }\end{array}$} & 2 & 0 & 430 & 37 & 0,00 & 0,00 \\
\hline & 4 & 0 & 342 & 28 & 0,00 & 0,00 \\
\hline & 1 & 0 & 383 & 23 & 0,00 & 0,00 \\
\hline & 3 & 1 & 333 & 19 & 0,30 & 5,26 \\
\hline & 5 & 2 & 329 & 18 & 0,61 & 11,11 \\
\hline & moyenne & 0,2 & 363,4 & 25 & 0,18 & 3,27 \\
\hline \multirow{6}{*}{$\begin{array}{l}2^{\mathrm{e}} \text { sec. } \\
\text { faibles }\end{array}$} & 7 & 3 & 448 & 39 & 0,67 & 7,69 \\
\hline & 10 & 2 & 334 & 25 & 0,60 & 8,00 \\
\hline & 8 & 4 & 410 & 22 & 0,98 & 18,18 \\
\hline & 6 & 5 & 301 & 25 & 1,66 & 20,00 \\
\hline & 9 & 7 & 422 & 34 & 1,66 & 20,59 \\
\hline & moyenne & 4,2 & 383 & 29 & 1,11 & 14,89 \\
\hline $5^{\mathrm{e}} \mathrm{sec}$ & 12 & 0 & 832 & 90 & 0,00 & 0,00 \\
\hline
\end{tabular}

forts 


\begin{tabular}{|c|c|c|c|c|c|}
\hline 14 & 0 & 550 & 52 & 0,00 & 0,00 \\
\hline 15 & 4 & 610 & 75 & 0,66 & 5,33 \\
\hline 11 & 3 & 478 & 52 & 0,63 & 7,77 \\
\hline 13 & 8 & 779 & 102 & 1,03 & 3,79 \\
\hline moyenne & 5 & 649,8 & 74,2 & 0,46 & 2,74 \\
\hline 17 & 2 & 559 & 73 & 0,36 & 4,08 \\
\hline faibles & 2 & 498 & 49 & 0,40 & 4,84 \\
\hline 19 & 3 & 540 & 62 & 0,56 & 5,33 \\
\hline 16 & 4 & 531 & 75 & 0,75 & 8,70 \\
\hline & 6 & 535 & 69 & 1,12 & 5,14 \\
\hline
\end{tabular}

On remarque d'abord que, lorsqu'il y a des erreurs, le pourcentage d'erreurs par contexte est toujours plus élevé que le N100. Chez les forts de $2^{\mathrm{e}}$ secondaire, trois élèves ne font aucune erreur de phrases complexes. L'élève 3 fait 0,3 erreur par 100 mots, ce qui correspond dans son cas à 5,25\% de contextes de phrases complexes, et l'élève 5 , qui a un N100 de 0,61 , fait erreur dans $11,11 \%$ des contextes. Chez les faibles de $2^{\mathrm{e}}$ secondaire, tous les élèves font des erreurs liées à la phrase complexe. Les élèves 6 et 9 font erreur dans $20 \%$ des contextes de phrases complexes, alors que leur N100 est de 1,66. L'élève 8 fait environ une erreur de phrase complexe par 100 mots, ce qui se révèle être une erreur dans $18 \%$ des contextes de phrases complexes. Deux autres élèves ( 7 et 10) font respectivement 3 erreurs dans 39 contextes (erreur dans $7,69 \%$ des contextes) et 2 erreurs dans 25 contextes (erreur dans $8 \%$ des contextes).

Chez les élèves de $5^{\mathrm{e}}$ secondaire, deux élèves forts ne font aucune erreur de phrase complexe. Les élèves 11 et 15 font respectivement 3 et 4 erreurs dans des textes contenant 52 et 75 contextes de phrases complexes, c'est-à-dire qu'ils font erreur dans un peu plus de $5 \%$ des contextes. L'élève 13 fait quant à lui erreur dans $7,84 \%$ des contextes. Tous les élèves faibles de $5^{\mathrm{e}}$ secondaire font des erreurs de phrases complexes. Il est intéressant de constater que leur taux d'erreurs par contexte est parfois plus faible que celui des élèves forts, variant entre $2,74 \%$ et $8,70 \%$.

On peut également voir que l'écart de 11,62 points qui existe entre les forts et les faibles en $2^{\mathrm{e}}$ secondaire est considérablement moindre en $5^{\mathrm{e}}$ secondaire (écart de 1,35 point). Il peut sembler surprenant de constater qu'il n'y a pas une énorme différence concernant le nombre d'erreurs liées à la phrase complexe entre les forts et les faibles de $5^{\mathrm{e}}$ secondaire, notamment parce que la différence entre eux pour toutes les catégories d'erreurs est plus marquée (voir fig. 1).

S'agissant des élèves forts, il apparait que ceux de $2^{\mathrm{e}}$ secondaire présentent en moyenne un plus faible pourcentage de contextes avec erreur $(3,27)$ que ceux de $5^{e}$ secondaire 
$(3,79)$. Le pourcentage de contextes avec erreurs indique donc de meilleures performances chez les plus jeunes, tout comme l'indiquaient les N100. On remarque toutefois que, pour presque deux fois plus de mots en moyenne en $5^{\mathrm{e}}$ secondaire $\left(363,4\right.$ mots en $2^{\mathrm{e}}$ secondaire et 649,8 en $5^{\mathrm{e}}$ secondaire), le pourcentage de contextes avec erreur indique une relative stabilité dans les performances des forts malgré des textes plus longs, un fait moins saillant avec les N100. Du côté des faibles, on remarque que le pourcentage moyen de contextes avec erreurs, tout comme la moyenne N100, est trois fois plus élevé en $2^{\mathrm{e}}$ secondaire $(14,89)$ qu'elle ne l'est en $5^{\mathrm{e}}$ secondaire $(5,14)$.

\section{Discussion}

50 Nous revenons ici sur certains résultats saillants concernant d'une part la composition syntaxique des textes et d'autre part la mesure des erreurs en fonction des contextes, pour les discuter de manière plus approfondie.

\subsection{Composition syntaxique}

51 L'analyse de la composition syntaxique des phrases graphiques (tableau 4) a révélé que le taux de phrases complexes est plus élevé chez les scripteurs plus âgés, qu'ils soient forts ou faibles, mais demeure le même ou presque entre les forts et les faibles d'un même niveau. Cela indique, dans la foulée des travaux de Hunt (1965) et de Paret $(1988,1991)$, que la présence de phrases complexes est un indicateur de maturité syntaxique, puisque ces dernières sont en proportion plus grande d'environ 20 points chez les élèves plus âgés. Paret observe aussi que les phrases simples sont « moins fréquentes avec l'âge : elles diminuent de $13 \%$ entre la $1^{\text {re }}\left[12\right.$ ans] et la $5^{\mathrm{e}}$ secondaires » (Paret, 1988, p. 187). Selon nos résultats, le taux de phrases simples chez les forts de $2^{\mathrm{e}}$ secondaire n'est pas plus bas que celui des faibles de $2^{\mathrm{e}}$ secondaire. Ainsi, dans l'hypothèse où les élèves forts jeunes feraient preuve d'une maturité syntaxique plus grande que leurs pairs plus faibles, cette maturité ne se manifeste pas par une proportion plus grande de phrases complexes, puisque, en pourcentage, les élèves faibles de $2^{\mathrm{e}}$ secondaire produisent légèrement plus de phrases complexes que les élèves forts de $2^{\mathrm{e}}$ secondaire (voir tableau 4). En revanche, comme nous le voyons dans le paragraphe qui suit, la proportion des phrases complexes formées par subordination distingue les élèves forts et faibles dès la $2^{\mathrm{e}}$ secondaire et s'avère un indicateur de maturité syntaxique chez les élèves de $2^{\mathrm{e}}$ secondaire.

52 L'analyse de la composition syntaxique des phrases complexes (voir tableau 5) a montré que la proportion de phrases juxtaposées ou coordonnées diminue au profit des phrases subordonnées entre la $2^{\mathrm{e}}$ et la $5^{\mathrm{e}}$ secondaire. Ces résultats rejoignent ceux de Paret (1988) qui indique qu' " au contraire [de l'enchâssement], la coordination de phrases diminue de $29 \%$, ce qui laisse à penser que la coordination est remplacée, en partie du moins, par l'enchâssement » (Paret, 1988, p. 188). Notre travail apporte un éclairage supplémentaire sur la maturité syntaxique en montrant en outre que la proportion de subordonnées (par opposition aux coordonnées ou juxtaposées) dans les phrases complexes distingue les forts et les faibles dès la $2^{\mathrm{e}}$ secondaire. Cela suggère que le taux de subordonnées est un indicateur d'une plus grande maturité syntaxique chez les élèves plus jeunes. En d'autres termes, le taux de subordonnées permet d'identifier des élèves plus matures au plan syntaxique dès un jeune âge, ce que ne permet pas le taux de phrases complexes. Les 
travaux antérieurs n'avaient pas comparé la maturité syntaxique des élèves à l'intérieur d'un même groupe d'âge.

\subsection{Taux d'erreurs par contexte}

53 Il convient de discuter de l'intérêt de la mesure du nombre d'erreurs par contexte. $\mathrm{Ce}$ taux permet de décrire et de comprendre plus précisément la maitrise de la phrase complexe (ou de tout autre objet grammatical) chez les élèves. Toutefois, selon nos résultats et contrairement à ce à quoi on aurait pu s'attendre, le nombre d'erreurs par contexte ne présente pas un intérêt plus grand que le nombre d'erreurs par cent mots : en effet, le nombre d'erreurs par contexte ne permet pas de différencier des élèves ou de détecter des problèmes qui n'auraient pas été détectés avec le nombre d'erreurs par cent mots. Nous n'avons cependant établi ce point qu'avec un petit nombre de textes et pour un seul objet grammatical. La question de l'intérêt du taux d'erreurs par contexte pourrait être investiguée pour d'autres objets grammaticaux.

Comme le souligne Paret (1988, p. 188), la composition syntaxique des textes, notamment la proportion de phrases simples et complexes de même que les différentes réalisations de la phrase complexe, est jusqu'à un certain point fonction des types de textes (narratifs, explicatifs, argumentatifs, etc.). Dans notre corpus, les textes des élèves de $2^{\mathrm{e}}$ secondaire étaient des textes descriptifs et ceux des élèves de $5^{\mathrm{e}}$ secondaire, des textes argumentatifs : en d'autres termes, ils n'étaient pas parfaitement comparables. Les résultats fournis par la mesure du nombre d'erreurs par contexte et ceux fournis par le N100 indiquent que la mesure du nombre d'erreurs par contexte n'a pas permis de mieux prendre en compte les éventuelles différences liées aux types de texte.

\section{Limites de cette recherche}

55 Le choix de déterminer si un élève est fort ou faible à l'aide des résultats pour l'ensemble des erreurs linguistiques constitue une première limite méthodologique. Ainsi, les élèves sélectionnés sont globalement forts ou globalement faibles, mais pas nécessairement forts ou faibles en syntaxe. Notre classement aurait peut-être été différent si on avait ordonné les élèves selon leurs résultats en syntaxe, ce qui aurait permis de mieux mettre en lumière ce qui différencie les forts et les faibles pour la maitrise des phrases complexes. Notons toutefois que les erreurs de syntaxe étant les plus nombreuses, le classement final risquait d'être similaire.

Notre description de la composition syntaxique des textes des élèves s'est limitée à certaines sous-catégories et a amalgamé dans la sous-catégorie « autres » les complétives, les incises, les incidentes, les subordonnées complément de phrases et les subordonnées adjointes. Ceci permettait de faire un lien direct avec le codage d'erreurs de Boivin et Pinsonneault $(2014,2017)$, mais limite la description de la maturité syntaxique des élèves de l'échantillon, telle que révélée par la composition de leurs textes. Il pourrait notamment être intéressant d'extraire les complétives pour en faire un code individuel, ce qui permettrait d'identifier de façon plus spécifique les problèmes liés à la maitrise de la complétive, par exemple le choix du mode et la concordance des temps. 
Dans un autre ordre d'idées, les différences notées entre les sous-groupes en termes de pourcentage d'erreurs n'ont pas été vérifiées à l'aide de méthodes statistiques; il nous est donc impossible pour l'instant d'affirmer qu'elles sont significatives.

\section{Conclusion}

En ce qui concerne la maturité syntaxique des élèves, les principales caractéristiques observées dans le corpus rejoignent celles des travaux antérieurs dans le domaine : le pourcentage de contextes de phrases complexes augmente avec l'âge, de même que le pourcentage de contextes de subordination dans les phrases complexes. À ces observations, nous ajoutons que le pourcentage de contextes de subordonnées dans les phrases complexes distingue les forts et les faibles en $2^{\mathrm{e}}$ secondaire ( 13 ans). Les forts de 2 ${ }^{\mathrm{e}}$ secondaire présentent un taux de subordination comparable à celui des élèves de $5^{\mathrm{e}}$ secondaire ( 16 ans, forts ou faibles) et semblent donc «matures » au plan syntaxique à cet égard. De façon générale, les forts de $2^{\mathrm{e}}$ et de $5^{\mathrm{e}}$ secondaire présentent des caractéristiques très similaires, pour la composition syntaxique et pour leurs performances liées aux phrases complexes.

Bien que la mesure des erreurs en termes de nombre d'erreurs par contexte permette une description plus fine de la maitrise des phrases complexes, elle ne constitue pas un outil de détection plus efficace des difficultés des élèves que le N100. La recherche pourrait poursuivre la comparaison entre les deux mesures pour d'autres objets grammaticaux.

Il nous semble que le concept de maturité syntaxique s'avère utile pour mieux comprendre les écrits des élèves, autant dans la composition syntaxique que dans les erreurs produites. Les connaissances sur la maturité syntaxique telles que celles présentées dans cet article nous semblent avoir des implications didactiques, notamment pour l'identification et la compréhension des difficultés des élèves, en particulier les plus jeunes et les plus faibles, et pour le soutien de leur développement scriptural.

\section{BIBLIOGRAPHIE}

BoIvin, Marie-Claude \& Pinsonneault, Reine. (2017, sous presse). Les erreurs de syntaxe, d'orthographe grammaticale et d'orthographe grammaticale des élèves québécois en contexte de production écrite. Revue canadienne de linguistique appliquée.

BoIvin, Marie-Claude \& PinsonneAult, Reine. (2014). Étude sur les erreurs de syntaxe, d'orthographe grammaticale et d'orthographe grammaticale des élèves québécois en contexte de production écrite. Rapport de recherche déposé au ministère de l'Éducation, du Loisir et du Sport du Québec, décembre 2014.

BoIvin, Marie-Claude \& PinsonNEAULT, Reine. (2008). La grammaire moderne : description et éléments pour sa didactique. Montréal, Canada : Beauchemin. 
CHARTRAND, Suzanne-Geneviève. (dir.) (1996). Pour un nouvel enseignement de la grammaire ( $2^{\mathrm{e}}$ éd.).

Montréal, Canada : Logiques.

DIEPE Groupe (1995). Savoir écrire au secondaire : étude comparative auprès de quatre populations francophones d'Europe et d'Amérique. Bruxelles, Belgique : De Boeck.

GOBBE, Roger. (1980). Pour appliquer la grammaire nouvelle. Paris, France : Duculot.

HUNT, Kellogg W. (1965). Grammatical Structures Written at Three Grade Levels (Rapport ${ }^{\circ} 3$ ).

Champaign, IL : NCTE.

LEFRANÇOIS, Pascale, LAURIER, Michel D., LAZURE, Roger \& Claing, Robert. (2008). Évaluation de

l'efficacité des mesures visant l'amélioration du français écrit du primaire à l'université. Montréal : Office québécois de la langue française.

LEMONNIER, France H. \& GAGNON, Odette. (2010). La qualité du français écrit : comment l'analyser?

Comment l'évaluer? Québec, Canada : Presses de l'Université Laval.

LIBERSAN, Lucie. (2003). Une grille de correction « nouvelle grammaire ». Correspondance, $8(3)$. <

www.ccdmd.qc.ca/correspo/Corr8-3/Grille.html>.

MANESSE, Danielle \& CogIS, Danièle. (2007). Orthographe : à qui la faute? Issy-les-Moulineaux, France : ESF.

MINISTÈRE DE L'ÉDUCATION, DU LOISIR ET DU SPORT. (2010). Évaluation de programme. Plan d'action pour l'amélioration du français. Premier rapport d'étape. Québec, Canada : Ministère de l'Éducation, du Loisir et du Sport.

NADEAU, Marie \& FISHER, Carole. (2006). La grammaire nouvelle : la comprendre et l'enseigner.

Montréal, Canada : Chenelière.

PARET, Marie-Christine. (1988). La syntaxe écrite des élèves du secondaire. Revue des sciences de l'éducation, 14(2), 183-204. <http://dx.doi.org/10.7202/900594ar>.

PARET, Marie-Christine. (1991). La syntaxe écrite des élèves du secondaire. Montréal, Canada : Faculté des sciences de l'éducation.

RieGEL, Martin, PeLlAT, Jean-Christophe \& Rioul, René. (2016). Grammaire méthodique du français (6 éd.). Paris, France : Presses universitaires de France.

RoY, Gérard-Raymond, LAFONTAINE, Lizanne \& LEGROS, Catherine. (1995). Le savoir grammatical après treize ans de formation. Sherbrooke, Canada : CRP.

\section{NOTES}

1. Ce travail a été financé en partie par le ministère de l'Éducation, du Loisir et du Sport du Québec, entente $\mathrm{n}^{\circ} 350017703$.

2. Les $2^{\mathrm{e}}$ et $5^{\mathrm{e}}$ années du secondaire correspondent respectivement à la $4^{\mathrm{e}}$ année du collège et à la $1^{\text {re }}$ année du lycée. Pour la suite du texte, nous réfèrerons généralement au niveau scolaire québécois.

3. Les phrases (18) à (23) plus loin constituent des exemples d'erreurs relevant de ces souscatégories.

4. Nous considérons la «concordance des temps » comme étant un phénomène d'ajustement du temps verbal dans une phrase complexe. Cet ajustement est déclenché par le temps du verbe de la phrase matrice. Il ne s'agit pas du choix du mode du verbe dans la phrase subordonnée, qui 
dépend de la catégorie sémantique du verbe qui commande la subordonnée (cf. Boivin \& Pinsonneault, 2008, p. 173) et fait l'objet d'un code distinct.

5. Les épreuves d'écriture consistent à rédiger un texte narratif ( $4^{\mathrm{e}}$ primaire), justificatif ( $6^{\mathrm{e}}$ primaire), informatif et justificatif ( $2^{\mathrm{e}}$ secondaire), et argumentatif $\left(5^{\mathrm{e}}\right.$ secondaire) sur un thème lié à l'histoire, la géographie ou la société québécoise. Le nombre de mots varie selon le niveau scolaire, mais les conditions de passation sont uniformes pour chaque niveau.

6. Nous avons fait le choix méthodologique d'opposer "élèves forts " et "élèves faibles » de manière à mettre en évidence d'éventuelles différences à l'intérieur d'un même niveau scolaire et de faire émerger les différences entre les niveaux scolaires. La maturité syntaxique impliquant l'idée de contraste, nous n'avons pas sélectionné d'élèves moyens.

7. Les exemples tirés des textes d'élèves conservent leurs erreurs.

8. Le nombre de phrases syntaxiques correspond à la somme du nombre de phrases simples et du nombre de contextes de phrases complexes.

9. Le nombre de contextes de phrases complexes est supérieur au nombre de phrases complexes du tableau 4, puisqu'une seule phrase complexe peut être formée à l'aide de plus d'un procédé de formation de phrase complexe, comme illustré en (17).

10. On peut se demander comment déterminer s'il y a une ou plusieurs erreurs. Dans le cas de l'exemple (20), nous considérons qu'il y a une erreur dans la construction de la corrélative, qui se manifeste par un placement erroné de tellement et de que.

\section{RÉSUMÉS}

Cet article explore la composition syntaxique des textes et la maitrise des phrases complexes chez les élèves québécois. L'analyse de 20 textes d'élèves montre que les élèves forts de $2^{\mathrm{e}}$ et de $5^{\mathrm{e}}$ secondaire (13 et 16 ans) présentent des caractéristiques similaires et la proportion de phrases complexes augmente avec le niveau scolaire. La proportion de phrases subordonnées distingue les forts des faibles de 13 ans. Le nombre d'erreurs de construction de phrases complexes est similaire chez les forts de 13 et de 16 ans et, chez les élèves faibles, il diminue remarquablement entre 13 et 16 ans. Le nombre moyen d'erreurs par 100 mots et le taux d'erreurs par contexte où l'erreur est possible sont des indicateurs comparables.

This article explores Quebec students' syntactic composition of texts and their ability to build complex sentences. The analysis of 20 texts shows that the high-achieving students aged 13 and 16 years old (8th and 11th grade) exhibit similar characteristics. The proportion of complex sentences raises with the students' age, and the proportion of subordinate clauses distinguishes the low-achieving from the high-achieving youngest students. The number of errors in the construction of complex sentences is similar for high-achieving students at both ages; it greatly diminishes in low-achieving students between age 13 and age 16. The average number of errors per 100 words and the rate of errors per context where an error is possible are comparable indicators. 
INDEX

Mots-clés : écriture, syntaxe, phrases complexes, erreurs, maturité syntaxique

Keywords : writing, syntax, complex sentences, errors, syntactic maturity

\section{AUTEURS}

MARIE-CLAUDE BOIVIN

Université de Montréal

KATRINE ROUSSEL

Université de Montréal

REINE PINSONNEAULT

Université du Québec à Montréal 